\title{
Effektorientierte Untersuchungen zur Algentoxizität fraktionierter Porenwässer und Eluate aus Elbesedimenten
}

\author{
Evelyn Claus $\cdot$ Helga Neumann-Hensel $\cdot$ Peter Heininger $\cdot$ Jan Schwarzbauer
}

Eingegangen: 11. März 2009/Akzeptiert: 29. April 2009/Online veröffentlicht: 26. Mai 2009

(C) Springer-Verlag 2009

\begin{abstract}
Zusammenfassung Hintergrund und Ziel der Studie Elbsedimente zeigen seit Jahren hohe Toxizitäten im Algenwachstumshemmtest, ohne deren stofflichen Hintergrund umfassend zu kennen. Daher wurde in einem Gemeinschaftsprojekt nach einer ersten screeningorientierten Analytik eine Wirkungsfraktionierung auf Basis einer Festphasenanreicherung mit nachfolgender sequentieller Elution entwickelt und zur wirkungsorientierten Analytik eingesetzt. Dadurch konnte neben Ammonium eine zweite organische Wirkkomponente isoliert werden, die sich durch eine hohe Lipophilie und Thermostabilität sowie niedrige Polarität und geringe Flüchtigkeit auszeichnet. Eine eindeutige chemische Identifizierung ist bislang nicht gelungen.

In einem zweiten Untersuchungsabschnitt wurde für die Algentoxizität in Elbsedimenten an zwei Standorten eine hohe örtliche Varianz sogar im kleinräumigen Maßstab belegt.
\end{abstract}

Verantwortliche Herausgeber: Jan Schwarzbauer · Peter Heininger · Evelyn Claus

J. Schwarzbauer $(\bowtie)$

Lehrstuhl für Geologie, Geochemie und Lagerstätten des Erdöls und der Kohle (LEK),

RWTH Aachen University,

Lochnerstraße 4-20, 52056 Aachen, Deutschland

E-Mail: schwarzbauer@lek.rwth-aachen.de

E. Claus $\cdot$ P. Heininger

Bundesanstalt für Gewässerkunde (BfG),

Abteilung G Qualitative Gewässerkunde

Am Mainzer Tor 1, 56068 Koblenz, Deutschland

H. Neumann-Hensel

Dr. Fintelmann und Dr. Meyer GmbH,

Chemische und biologische Handelslaboratorien,

Mendelssohnstraße 15, 22761 Hamburg, Deutschland
Schlüsselwörter Effektdirigierte Analyse $\cdot$ Phytotoxizität · Algenwachstumshemmtest · Organische Schadstoffe · Elbe $\cdot$ Sedimente $\cdot$ SPE-Methode

\section{Phytotoxicity of Elbe river sediments}

Abstract Background and aim Sediments of the Elbe river exhibit over the last years extended toxicities as revealed by the algae growth inhibition test. However these ecotoxicological effects cannot be linked directly to discrete pollutants. Therefore, this study dealt not only with GC/MS based screening analyses to identify these components, but, more important, with an effect-directed analytical approach based on solid phase extraction followed by a sequential elution procedure. Applying this method a second component (beside ammonium as active substance) was isolated, that can be characterized to be lipophilic, low volatile, thermostable and nonpolar. However, a structural identification of this component has failed so far.

In a second part of the study a high variation of the algae toxicity was observed with respect to the spatial distribution on a very small scale.

Keywords Effect-directed analysis $\cdot$ Phytotoxicity $\cdot$ Algae growth inhibition test - Organic pollutants $\cdot$ Elbe river .

Sediments $\cdot$ SPE Method

\section{Problemstellung}

Gewässergüte und entsprechende Gewässerbelastungen werden anhand physikalischer, chemischer, biologischer und ökotoxikologischer Kriterien erfasst. Da physikalische und chemische Daten per se keine direkte Aussage über das 
Wirkungspotenzial liefern, werden zunehmend ökotoxikologische Verfahren zur Gefährdungsabschätzung eingesetzt. Besonders durch die Arbeiten von Chapman (1986) zur Sedimenttriade erlangten auch ökotoxikologische und biologische Untersuchungen eine Bedeutung in der Gewässerbewertung.

Ökotoxikologische Testsysteme beziehen durch ihre Wirkmechanismen auch Schadstoffe ein, die nicht durch die chemische Analytik erfasst werden, aber dennoch in wirkungsrelevanten Konzentrationen bioverfügbar sind. Problematisch für Maßnahmen zur Prävention oder Reduktion von festgestellten Belastungen ist der Umstand, dass sich gemessene ökotoxikologische Effekte häufig nicht auf stoffliche Einzelkomponenten zurückführen lassen. Daher wurde seit Beginn der 1990er-Jahre weltweit verstärkt die Methodik der wirkungsbezogenen Analytik besonders auch in Sedimenten entwickelt und eingesetzt. Biotestpaletten mit diversen Endpunkten und Trophieebenen wurden zusammengestellt, die oftmals auch einen Algenwachstumshemmtest in Sedimentextrakten, -eluaten oder -porenwässern enthielten (Okamura et al. 1996; Ahlf et al. 2002), wobei ein wesentlicher Bestandteil der wirkungsorientierten Analytik die verschiedenen Methoden der Fraktionierung von Sedimentextrakten bzw. die Anreicherung von Wasserproben wurden.

Ein sensitiver Test in der Gewässergütebewertung ist der Algenwachstumshemmtest, der im sedimentären Bereich an Eluaten und Porenwässern durchgeführt wird. Bisherige Ansätze zur wirkungsorientierten Analytik haben sich im Wesentlichen jedoch auf die Sedimentextrakte konzentriert, bislang also die eigentlichen Testgüter nicht direkt in die Analytik einbezogen. Dennoch konnten eine Reihe von Kontaminanten und Einflussfaktoren auf die Toxizität im Algenwachstumshemmtest in Gewässern und auch in Böden identifiziert werden. Aus stofflicher Sicht sind neben Polyzyklischen Aromatischen Kohlenwasserstoffen (PAK) u. a. Herbizide, ausgewählte Heterozyklen sowie Ammoniumionen als Verursacher bekannt. Darüber hinaus wurden aber auch natürliche Faktoren wie die Lichtintensität, der pH-Wert und das Redoxpotenzial als Einflussgrößen auf den Algenwachstumshemmtest erkannt (Baun et al. 2002; Grote et al. 2005a; Wahrendorf et al. 2005; Christensen et al. 2006; Brack et al. 2007; Eisenträger et al. 2008; Källquist et al. 2008).

Eine besondere Situation mit Bezug zur ökotoxikologischen Bewertung von Sedimenten ist an der Elbe festzustellen. Trenduntersuchungen von 1991-2001 (Heininger et al. 2003) belegten den Rückgang der Schwermetallbelastung, während organische Schadstoffe keinen einheitlichen Trend aufwiesen. Demgegenüber aber zeigten Algentoxizitäten einen steigenden Trend. So übertrafen die Ergebnisse des Algenwachstumshemmtests von 2001 an ausgewählten Messstellen die Daten von 1992. Diese Entwicklung zu eher erhöhten Algentoxizitäten setzt sich bis heute fort, wobei insbesondere das Jahr 2007 hohe Toxizitäten im gesamten Elbeverlauf aufwies. Daher wurden zahlreiche Untersuchungen zur Sedimentbewertung unter Einbeziehung des Algenwachstumshemmtests und einer wirkungsorientierten Analytik durchgeführt (Brack et al. 2007; Schwab 2008). Unter anderem wurden nach dem Elbehochwasser 2002 in fraktionierten Sedimentextrakten im Längsverlauf Algen als Testsystem eingesetzt (Grote et al. 2005b). Alle Proben zeigten mehr oder weniger hohe toxische Effekte mit Maximalwerten in Königstein, Wittenberg, Barby, Magdeburg und im Spittelwasser. Hsu et al. (2007) betrachteten die saisonale Anhängigkeit der Algentoxizität an Proben aus der Elbe (Nähe Hamburg) und der Dommel (Nähe Eindhoven). Diese Untersuchungen wurden sowohl in Wasserproben als auch in Eluaten von Sedimenten verschiedener Entnahmetiefen durchgeführt. Einen großen Einfluss auf die Höhe der gemessenen Toxizitäten im saisonalen Verlauf hatten hydrologische Veränderungen und die Algenblüte im Untersuchungszeitraum. Am Elbestandort wurden in den Wasserproben höhere Toxizitäten als in oberen Sedimentschichten gefunden. Tiefere Sedimentschichten zeigten eine noch geringere Toxizität. Eine genaue stoffliche Zuordnung der gemessenen ökotoxikologischen Effekte zu stofflichen Ursachen konnte bislang nicht getroffen werden.

\section{Ziel}

Da die ökotoxikologischen Untersuchungen von Elbsedimenten seit Jahren hohe Toxizitäten im Algenwachstumshemmtest (DIN 38412 L33) zeigen, ohne deren stofflichen Hintergrund umfassend zu kennen, werden in einem Gemeinschaftsprojekt der Bundesanstalt für Gewässerkunde, der RWTH Aachen und Dr. Fintelmann und Dr. Meyer $\mathrm{GmbH}$ sowohl wirkungsorientierte Screening-Analysen als auch die Entwicklung einer SPE-basierten (SPE: solid phase extraction) Wirkungsfraktionierung durchgeführt. Ziel ist eine Eingrenzung und chemische Charakterisierung der maßgeblichen Wirkkomponente sowie die Charakterisierung der Toxizität in räumlicher und zeitlicher Entwicklung. Dabei wird insbesondere das eigentliche Testgut, also Porenwässer und Eluate, in den Fokus der Untersuchung gestellt.

\section{Ergebnisse und Diskussion}

Die bisherigen Untersuchungen gliedern sich in drei Teilabschnitte. Neben einer ersten screeningorientierten Analytik wurde eine Wirkungsfraktionierung auf Basis einer Festphasenanreicherung mit nachfolgender sequentieller Elution entwickelt und zur wirkungsorientierten Analytik 
eingesetzt. Zusätzlich wurde auch eine Erfassung der zeitlichen und besonders räumlichen Varianz der im Elbesystem auftretenden Algentoxizität durchgeführt. Nachfolgend werden die gemessenenen Algentoxizitäten in pT-Werten angegeben. (potentia Toxicologiae $=$ toxikologischer Exponent $=$ negativer binärer Logarithmus des ersten nicht mehr toxischen Verdünnungsfaktors in einer Verdünnungsreihe mit dem Verdünnungsfaktor 2 (Krebs 1988, 2000)).

\subsection{Analytik von Porenwasser und Eluat}

Es ist bereits bekannt, dass die in den Elbesedimenten detektierte Algentoxizität partiell durch erhöhte Ammoniumkonzentrationen verursacht wird. Diese Stoffkomponente erklärt jedoch nicht die Gesamttoxizität. Daher wurden zur Identifizierung einer zweiten, organischen Stoffkomponente zunächst als vorbereitender Schritt in einem Nontarget-screening-Ansatz mit GC/MS-Analytik Eluate und Porenwässer von Elbesedimenten untersucht. Aus dem erhaltenen breiten Spektrum an organischen Inhaltsstoffen wurden durch Vergleich mit zeitgleich detektierten Stoffspektren von Sedimenten der Donau und des Rheins eine Gruppe von elbetypischen Porenwasser- und EluatInhaltsstoffen (N-Phenyl-2-naphthylamin, Tetrabutylzinn, Bis(chlorpropyl)ether, HCHs, DDA, Dichlorbenzole, Hexachlorbenzol, Alachlor, Lenacil) selektiert. Diese Stoffe wurden dann zur zielgerichteten Analytik an zwei ausgewählten Sedimenten eingesetzt. Untersucht wurden Porenwässer und Eluate von sedimentärem Material aus dem Mittellauf der Elbe (Lauenburg und Magdeburg), die deutliche Effekte im Algenwachstumshemmtest mit Desmodesmus subspicatus (pT-Werte 2 bis 5) zeigten. Überraschenderweise konnte keiner der ausgewählten Schadstoffe in den untersuchten Proben detektiert und somit die zweite Wirkkomponente nicht identifiziert werden.

\subsection{Effektdirigierte Analytik auf Basis einer SPE-Fraktionierung}

Da der zielgerichtete Screeningansatz keine zweite Wirkkomponente eindeutig isolieren konnte, wurde entsprechend in einem Folgeschritt eine Fraktionierung der Eluat- und Porenwasserextrakte desselben Probenkontingents auf Basis einer Festphasenanreicherung (SPE) mit nachfolgender sequentieller Elution (Abb. 1) durchgeführt und in den einzelnen Fraktionen die Algenwachstumshemmung getestet.

Die Trennung der organischen Anteile erzeugte Fraktionen mit aufsteigender Lipophilie bzw. abnehmender Polarität der Inhaltsstoffe. Toxizitäten konnten in den unpolaren Fraktionen (S4 und S5) festgestellt werden, dies besonders ausgeprägt in Porenwasser und Eluat der Probe aus dem Bereich Magdeburg (Tabelle 1). Die toxischen Fraktionen wurden nachfolgend einem Non-target-Screening zugeführt,

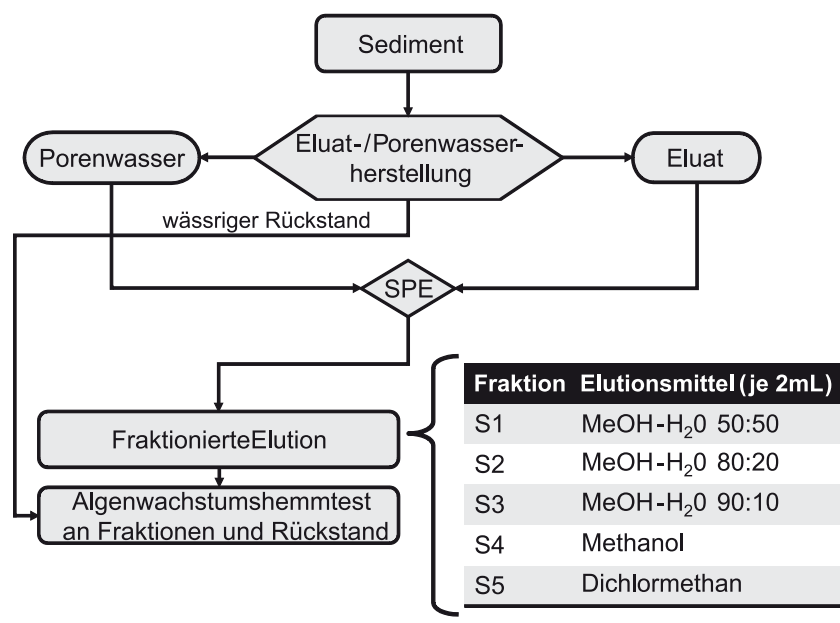

Abb. 1 Flussschema zur wirkungsorientierten Analytik von Porenwässern und Eluaten auf Basis einer Festphasenanreicherung mit nachfolgender sequentieller Elution

aber auch hier konnte auf Basis von GC/MS-Analysen keine eindeutige Wirkkomponente identifiziert werden.

Die dargestellte Wirkungsfraktionierung konnte in einem zweiten Ansatz mit Proben aus dem Hamburger Hafen, die ebenfalls hohe Algentoxizitäten aufwiesen (pT 5-6 im Porenwasser), reproduziert und die Methodik somit validiert werden. Interessanterweise zeigten sich bei beiden Probenkontingenten auch in den extrahierten wässrigen Rückständen teilweise eine erhöhte Toxizität (bis pT 6). Als Erklärung dieses Phänomens können Demaskierungseffekte von toxischen anorganischen oder sehr polaren Substanzen durch die Abtrennung lipophilen organischen Materials angenommen werden.

Ein zweiter bemerkenswerter Effekt war ebenfalls bei der Bestimmung der Wiederholpräzision zu beobachten. Ein Vergleich der Ergebnisse von unfiltrierten Proben mit filtriertem Probenmaterial (Porenwasser/Eluat) zeigte eine deutliche Erniedrigung der fraktionierbaren Toxizität durch die Filtration. Da diese Wirkkomponente lipophilen Charakter hat, ist

Tabelle 1 Gemessene Hemmungen (in \%) im Algenwachstumshemmtest in Anlehnung an DIN 38412 L33 in der ersten Verdünnungsstufe nach Fraktionierung (negative Werte indizieren eine Wachstumsförderung)

\begin{tabular}{|l|c|c|}
$\begin{array}{l}\text { Magdeburg } \\
\text { Hemmung in G1 }\end{array}$ & $\begin{array}{c}\text { PW } \\
(\mathbf{3 2} \%)\end{array}$ & Eluat \\
\hline S1 & -37.4 & 3.5 \\
\hline S2 & -77.1 & -84.5 \\
\hline S3 & -29.8 & 28.6 \\
\hline S4 & 49.3 & 67.3 \\
\hline S5 & 99.3 & 59.2 \\
\hline
\end{tabular}




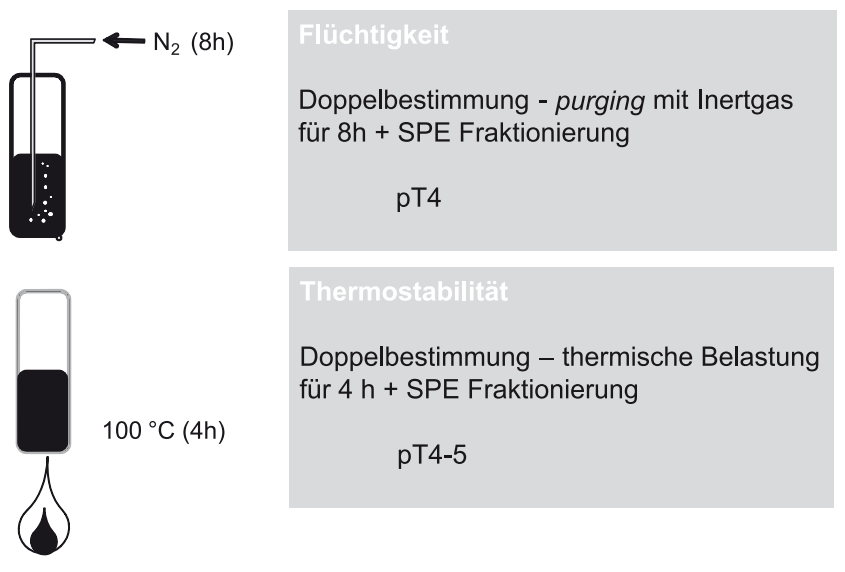

Abb. 2 Experimentelle Ansätze zur Charakterisierung der Flüchtigkeit und der Thermostabilität einer fraktionierbaren Wirkungskomponente in Eluaten und Porenwässern

die Assoziation mit partikulärem Material wahrscheinlich, und somit deutet die Toxizitätsreduktion durch Filtration auf einen partikelassoziierten Anteil der Algentoxizität hin.

Die Methodik der SPE-basierten Wirkungsfraktionierung wurde auch genutzt, um indirekt Hinweise auf die physikochemischen Eigenschaften der zweiten Wirkkomponente zu erhalten. In zwei verschiedenen experimentellen Ansätzen (Abb. 2) wurden die Thermostabilität und die Flüchtigkeit untersucht. Da auch nach intensivem Ausblasen des Untersuchungsmaterials und nach thermischer Belastung keine Reduktion der Toxizität in den unpolaren Fraktionen erkennbar war, kann die zweite Wirkkomponente als thermostabil und nicht leichtflüchtig charakterisiert werden.

\subsection{Räumliche Verteilung der Phytotoxizität}

An zwei Standorten an der Elbe (Leopoldshafen bei Dessau und Buhnenfelder bei Lauenburg) wurde in jeweils kleinräumigem Maßstab die Varianz der Ergebnisse des Algenwachstumshemmtests getestet. Dazu wurden an jeweils drei Lokalitäten auf einer Gesamtlänge von ca. $200 \mathrm{~m}$ je 4 Sedimentproben im Abstand von ca. $2 \mathrm{~m}$ entnommen und die Algentoxizität der korrespondierenden Eluate und Porenwässer gemessen. Sowohl zwischen den einzelnen Probenahmestandorten auf einer Gesamtlänge von ca. $200 \mathrm{~m}$ als auch im kleinräumigen Maßstab von ca. $2 \mathrm{~m}$ schwanken die Algenwachstumshemmwerte stark. Diese sehr hohe örtliche Varianz ist in Tabelle 2 für den Standort Leopoldshafen (Dessau) illustriert. Aber auch einige sedimentologische
Parameter, wie z.B. die Gehalte an organischem Kohlenstoff (TOC) und die Korngröße, variieren stark. So liegt der TOC-Gehalt für Dessau zwischen 2,7 und 8,3\%, in Lauenburg zwischen 0,7 und 4,8\%. Der Anteil der Feinkornfraktion $<20 \mu \mathrm{m}$ liegt in Dessau zwischen 2,4 und $84 \%$, in Lauenburg zwischen 10 und $59 \%$. Es lässt sich jedoch keine Korrelation zwischen den sedimentologischen Daten und den gemessenen ökotoxikologischen Effekten erkennen.

Die zeitliche Abhängigkeit der Algenwachstumshemmung in fraktionierten Eluaten und Porenwässern wurde am Standort Dessau über einen Zeitraum von bisher acht Monaten getestet. Eine hohe Algentoxizität, wie sie für diesen Standort noch 2007 typisch war (pT 5 für das Porenwasser, pT 3 für das Eluat), konnte in den vergangenen Monaten nicht bestätigt werden.

\section{Ausblick}

Die Anwendung einer wirkungsorientierten Analytik von Sedimentporenwässern und -eluaten auf Basis einer SPEAnreicherung mit nachfolgender sequentieller Elution hat folgenden aktuellen Kenntnisstand zur Algentoxizität in Elbesedimenten erbracht:

- Ammoniumionen sind eine Wirkkomponente für Algentoxizität in Elbesedimenten. Die gemessenen Effekte lassen sich aber nicht allein auf diese reduzieren.

- Die zweite Wirkkomponente, die durch SPE ,fraktionierbar" ist, sollte organischen Charakters sein. Sie zeichnet sich durch hohe Lipophilie und Thermostabilität sowie niedrige Polarität und geringe Flüchtigkeit aus.

- Räumliche und zeitliche Variationen der Algentoxizität schwanken im Elbegebiet erheblich, ein ursächlicher Zusammenhang mit den ermittelten sedimentologischen Eigenschaften ist nicht erkennbar.

\section{Literatur}

Ahlf W, Hollert H, Neumann-Hensel H, Ricking M (2002) A Guidance for the assessment and evaluation of sediment quality. J Soils Sediments (2):37-42

Baun A, Justesen KB, Nyholm N (2002) Algal test with soil suspensions and elutriates: A comparative evaluation for PAH-contaminated soils. Chemosphere 46:251-258

Brack W, Klamer HJC, de Alda ML, Barcelo D (2007) Effect-directed analysis of key toxicants in European river basins - a review. Environ Sci Pollut Res 14(1):30-38

Chapman PM (1986) Sediment quality criteria from the sediment quality triad: an example. Environ Toxicol Chem 5:957-964
Tabelle 2 Erfasste Algentoxizitäten (pT-Werte) in einem räumlichen Raster im Leopoldshafen bei Dessau

\begin{tabular}{l|cccc|ccccc|cccc}
\hline Leopoldshafen Dessau & \multicolumn{3}{c}{ Lokalität 1 } & \multicolumn{3}{c}{ Lokalität 2 } & \multicolumn{3}{c}{ Lokalität 3 } \\
\hline Porenwasser & 4 & 1 & 1 & 1 & 1 & 4 & 1 & 4 & 1 & 1 & 1 & 1 \\
Eluat & 5 & 1 & 5 & 4 & 1 & 6 & 5 & 5 & 6 & 5 & 1 & 5 \\
\hline
\end{tabular}


Christensen AM, Nakajima F, Baun A (2006) Toxicity of water and sediment in a small urban river (Store Vejlea, Denmark). Environ Pollut 144:621-625

DIN 38412 L33 Deutsche Einheitsverfahren zur Wasser-, Abwasserund Schlammuntersuchung; Testverfahren mit Wasserorganismen (Gruppe L); Bestimmung der nicht giftigen Wirkung von Abwasser gegenüber Grünalgen (Scenedesmus-Chlorophyll-Fluoreszenztest) über Verdünnungsstufen (L 33)

Eisenträger A, .Brinkmann C, Hollert H, Sagner A, Tiehm A, Neuwoehner J (2008) Heterocyclic compounds: Toxic effects using algae, daphnids, and the Salmonella/microsome test taking methodical quantitative aspects into account. Environ Toxicol Chem 27(7):1590-1596

Grote M, Brack W, Walter HA, Altenburger R (2005a) Light as a confounding factor for toxicity assessment of complex contaminated sediments. Environ Toxicol Chem 24(12):3143-3152

Grote M, Altenburger R, Brack W, Moschutz S, Mothes S, Michael C, Narten G-B, Paschke A, Schirmer K, Walter H, Wennrich R, Wenzel K-D, Schüürmann G (2005b) Ecotoxicological profiling of transect river Elbe sediments. Acta hydrochim hydrobiol 33:555-569

Heininger P, Pelzer J, Claus E, Pfitzner S (2003) Results of long-term sediment quality studies on the river Elbe. Acta hydrochim hydrobiol 31(4/5):356-367
Hsu P, Matthai A, Heise S, Ahlf W (2007) Seasonal variation of sediment toxicity in the Rivers Dommel and Elbe. Environ Pollut 148(3):817-823

Källquist T, Milacic R, Smital T, Thomas KV, Vranes S, Tollefsen KE (2008) Chronic toxicity of the Sava River sediments and river water to the algae Pseudokirchneriella subcapitata. Water Res 42:2146-2156

Krebs F (1988) Der pT-Wert: ein gewässertoxikologischer Klassifizierungsmaßstab (The pT-value as a classification index in aquatic toxicology). GIT Fachzeitschr Laboratorium 32:293-296

Krebs F (2000) Ökotoxikologische Bewertung von Baggergut aus Bundeswasserstraßen mit Hilfe der pT-Wert-Methode. Hydrologie Wasserbewirtschaftung 44:301-307

Okamura H, Luo R, Aoyama I, Liu D (1996) Ecotoxicity assesment of the aquatic environment around Lake Kojima, Japan. Environ. Toxicol Wat Qual 11:213-221

Schwab K (2008) Effect-directed identification of bioavailable toxic organic compounds in contaminated sediments. Dissertation, Martin-Luther-Universität Halle-Wittenberg

Wahrendorf DS, Krebs F, Manz W (2005) Wirkung von AmmoniumStickstoff auf den Wachstumshemmtest mit der Grünalge Desmodesmus subspicatus nach DIN 38412-L33: BfG-Bericht 1468, Koblenz 